\title{
PERFIL DOS CONSELHEIROS E SEU CONHECIMENTO EM RELAÇÃO AO CONSELHO MUNICIPAL DE SAÚDE: REVISÃO INTEGRATIVA
}

\author{
PROFILE OF DIRECTORS AND ITS KNOWLEDGE IN RELATION TO THE \\ MUNICIPAL HEALTH COUNCIL: INTEGRATION REVIEW
}

\author{
Fernanda Souza Matos, Rafael da Silva Passos, Eduardo Nagib Boery, Alba Benemérita Alves \\ Vilela, Adriana Alves Nery, Ismar Eduardo Martins-Filho
}

Universidade Estadual do Sudoeste da Bahia - UESB

\begin{abstract}
In order to promote social participation in the process of the development of Health Policies, increasing democracy and promoting policies and actions that contemplate the community is the creation of the Municipal Health Council. Aim of this study was to present an integrative review on the profile of counselors and their knowledge and qualification in relation to the Municipal Health Council. This is an integrative review carried out in the Virtual Health Library (VHL) and Scientific Electronic Library Online (SciElo) databases. We found 7 articles that contemplated the proposed objective. Counselors are composed mostly of males, older adults, and full-time higher education. Most of them perceived a lack of qualification and qualification of the Health Counselors regarding the structure of the SUS and its role, demonstrating a fragility in the offer by managers and social representatives.
\end{abstract}

Keywords: Health Councils, Social control, Health Policy.
Resumo

Com o intuito de promover a participação social no processo do desenvolvimento das Políticas de Saúde, aumentando a democracia e promovendo as políticas $e$ ações que contemplem a comunidade é que foi criado o Conselho Municipal de Saúde. Objetivou-se neste estudo apresentar uma revisão integrativa sobre o perfil dos conselheiros $e$ seu conhecimento $e$ capacitação em relação ao Conselho Municipal de Saúde. Trata-se de uma revisão integrativa realizada nas bases de dados Biblioteca Virtual em Saúde (BVS) e Scientific Electronic Library Online (SciElo). Foram encontrados 7 artigos que contemplaram o objetivo proposto. Os Conselheiros são compostos em sua maioria pelo sexo masculino, adultos mais velhos e com ensino superior completo. Em sua maioria percebeu-se falta de qualificação e capacitação dos Conselheiros de Saúde quanto a estrutura do SUS e o seu papel, demonstrando uma fragilidade na oferta pelos gestores e representantes sociais.

Palavras-chave: Conselhos de Saúde, Controle social, Políticas de Saúde. 
Introdução

A criação do Sistema Único de Saúde (SUS), na década de 1980 , foi através de várias lutas e conquistas vivenciadas na área política e social no Brasil. A partir desse período, ocorreram diversos avanços nas políticas de saúde. Dentre estes, a participação da comunidade com a incorporação de novos atores sociais no processo decisório repercutiu de forma positiva para o fortalecimento da democracia ${ }^{1}$.

A participação da comunidade é garantida pela Lei no 8.142/1990, tendo como instâncias colegiadas os conselhos de saúde. Compostos por representantes do governo, prestadores de serviço, profissionais de saúde e usuários, são responsáveis por estratégias e pelo controle da execução da política de saúde, inclusive no que se refere aos aspectos econômicos e financeiros ${ }^{2}$.

Neste contexto, o Conselho Municipal de Saúde (CMS) tem competência para fiscalizar, planejar, propor e controlar os recursos da área de saúde no orçamento municipal, podendo e devendo, ainda, atuar na elaboração do Plano Plurianual de Saúde. O controle, o planejamento e a fiscalização do Fundo Municipal de Saúde, para onde são destinados os recursos a serem gastos com a saúde no município, também competem ao $\mathrm{CMS}^{3}$. Desde modo, na perspectiva de fortalecer o exercício do controle social na política de saúde, faz-se necessário, por parte dos sujeitos políticos envolvidos no processo, conhecer efetivamente o SUS, a legislação, a realidade epidemiológica, assistencial, financeira, e política, visando o acompanhamento e avaliação do sistema de informações em saúde nos níveis municipal, estadual e federal ${ }^{4,5}$.

Diante disso, ressalta-se a importância de realizar uma revisão integrativa que apresente o perfil e conhecimento dos conselheiros municipais de saúde. Assim, este estudo tem como objetivo apresentar uma revisão integrativa sobre o perfil dos conselheiros e seu conhecimento e capacitação em relação ao Conselho Municipal de Saúde.

\section{Metodologia}

Trata-se de uma revisão integrativa, baseada na leitura compreensiva de artigos relacionados com o tema. A busca foi realizada através de bases de dados existentes Biblioteca Virtual em Saúde (BVS) e Scientific Electronic Library Online (SciElo), utilizando as seguintes palavras chaves: conselhos de saúde, controle social e políticas de saúde. Os critérios de inclusão adotados foram: artigos publicados em inglês, espanhol e português, artigos disponíveis nas bases de dados escolhidas. Como critérios de exclusão definiu-se os artigos que não tinham relação com o tema referido.

$\mathrm{Na}$ base de dados BVS foram encontrados duzentos e seis artigos sobre conselheiros de saúde, sendo selecionados três artigos para análise, por atender os critérios de inclusão. $\mathrm{Na}$ base de dados SciELO, foram encontrados duzentos e oitenta artigos sobre conselhos de saúde, foram selecionados quatro estudos para compor a revisão.

Após a análise dos artigos, foi realizada a síntese dos dados, contemplando, objetivo, metodologia, resultados, discussão e conclusão.

\section{Resultados e Discussões}

A amostra final de sete artigos foi alcançada após análise inicial de alguns artigos obtidos através da base de dados BVS e SciELO. A maior parte dos artigos selecionados foi publicada em 2016 (com dois artigos publicados) e 2009 (com dois artigos publicados) seguido de 2012, 2013 e 2017 (com um artigo publicado em cada ano).

Tabela 1. Distribuição dos artigos incluídos na revisão integrativa, segundo objetivo e métodos empregados, 2017.

\begin{tabular}{|c|c|c|}
\hline Artigos & Objetivos & Métodos \\
\hline FERNANDES 2017 & $\begin{array}{l}\text { Conhecer a percepção dos conselheiros } \\
\text { municipais de saúde em relação ao } \\
\text { acesso à informação de saúde e } \\
\text { inclusão digital de um município de } \\
\text { porte médio do interior paulista. }\end{array}$ & $\begin{array}{l}\text { Trata-se de pesquisa qualitativa, } \\
\text { alicerçada no método de estudo de caso, } \\
\text { realizada com } 16 \text { conselheiros titulares } \\
\text { de saúde. Foi realizada entrevista } \\
\text { orientada por roteiro semiestruturado e } \\
\text { os dados foram analisados à luz da } \\
\text { análise de conteúdo representacional } \\
\text { temática. }\end{array}$ \\
\hline
\end{tabular}

Continua... 


\begin{tabular}{|c|c|c|}
\hline LOPES 2016 & $\begin{array}{l}\text { Compreender o funcionamento e as } \\
\text { contribuições do Conselho Municipal } \\
\text { de Saúde de Belo Horizonte para a } \\
\text { inserção da sociedade nos processos } \\
\text { políticos. }\end{array}$ & $\begin{array}{l}\text { Estudo qualitativo, os dados coletados } \\
\text { são de origem primária, tendo como } \\
\text { base entrevistas semiestruturadas } \\
\text { realizadas com os membros titulares do } \\
\text { Conselho Municipal de Saúde de Belo } \\
\text { Horizonte. }\end{array}$ \\
\hline LEMOS 2016 & $\begin{array}{l}\text { Compreender aspectos do perfil dos } \\
\text { conselheiros municipais de saúde de } \\
\text { Anápolis-GO e sua visão sobre sua } \\
\text { atuação no Conselho Municipal de } \\
\text { Saúde de Anápolis (CMSA). }\end{array}$ & $\begin{array}{l}\text { O método de abordagem foi quantitativo } \\
\text { e qualitativo, e a ferramenta de pesquisa } \\
\text { foi a aplicação de questionário } \\
\text { estruturado com } 14 \text { questões, sendo } 10 \\
\text { fechadas e quatro abertas, para os } \\
\text { conselheiros municipais de saúde ativos }\end{array}$ \\
\hline SOUZA, et al., 2013 & $\begin{array}{l}\text { Analisar o perfil dos Conselheiros de } \\
\text { Saúde do Município de Jequié-BA e } \\
\text { identificar seu conhecimento acerca da } \\
\text { função. }\end{array}$ & $\begin{array}{l}\text { Realizou-se uma pesquisa qualitativa, } \\
\text { descritiva e exploratória, realizada com } \\
\text { dez Conselheiros de Saúde, atuantes em } \\
2008 \text {. }\end{array}$ \\
\hline COTTA, 2009 & $\begin{array}{l}\text { Analisar o nível de informação e } \\
\text { conhecimento dos Conselheiros } \\
\text { Municipais de Saúde de Viçosa-MG, } \\
\text { em relação a suas atribuições e } \\
\text { funções. }\end{array}$ & $\begin{array}{l}\text { Trata-se de um estudo transversal, cujos } \\
\text { elementos de análise foram: entrevistas } \\
\text { semiestruturadas, realizadas por um } \\
\text { único entrevistador devidamente } \\
\text { treinado, durante o período de abril a } \\
\text { julho de } 2006 \text { e observação direta, não } \\
\text { participante, das reuniões do Conselho } \\
\text { Municipal de Saúde (CMS). As entrevistas } \\
\text { foram gravadas depois da autorização } \\
\text { dos entrevistados, sendo posteriormente } \\
\text { transcritas. }\end{array}$ \\
\hline RAMOS 2012 & $\begin{array}{l}\text { Apresentar o perfil dos conselheiros de } \\
\text { quatro conselhos setoriais do } \\
\text { Município de Santo André }\end{array}$ & $\begin{array}{l}\text { O levantamento dos dados foi feito } \\
\text { através de entrevistas fechadas para } \\
\text { identificação do perfil do entrevistado. A } \\
\text { amostra de pesquisa englobou } 97 \\
\text { conselheiros ( } 48 \text { conselheiros do poder } \\
\text { público e } 49 \text { conselheiros da sociedade } \\
\text { civil) de quatro conselhos municipais. }\end{array}$ \\
\hline SALIBA 2009 & $\begin{array}{l}\text { Conhecer o perfil dos conselheiros e a } \\
\text { participação destes nos processos } \\
\text { decisórios e nas ações concretas } \\
\text { relacionadas à gestão em saúde e o } \\
\text { impacto do curso de capacitação. }\end{array}$ & $\begin{array}{l}\text { Foi realizado um estudo exploratório } \\
\text { sobre cada Conselho Municipal de Saúde, } \\
\text { por meio de análise de documentos e } \\
\text { entrevistas preliminares com os } \\
\text { presidentes dos conselhos municipais de } \\
\text { saúde. }\end{array}$ \\
\hline
\end{tabular}

Em relação à Tabela 1, no que se referem aos objetivos propostos pelos estudos verifica-se que os mesmos buscam o perfil dos conselheiros, conhecer o nível de conhecimento e capacitação dos Conselheiros Municipais de Saúde. Em relação ao delineamento da pesquisa, identificou-se que das sete publicações, dois utilizaram abordagem quantitativa, três qualitativa e dois quantitativo e qualitativo.

A maioria dos estudos encontrados foram desenvolvidos no estado de São Paulo (3), e Minas Gerais (2), seguido de Goiás (1) e Bahia (1). A análise dos artigos permitiu a identificação de unidades temáticas presentes nas discussões sobre perfil e conhecimento dos conselheiros municipais de saúde.

\section{Perfil do Conselheiro Municipal de Saúde}

O perfil dos representantes do conselho de saúde, de acordo com a análise dos estudos da tabela 1, identificou-se que a maioria era do sexo masculino ${ }^{6-8}$. Um estudo encontrou que a proporção de homens e mulheres são semelhantes ${ }^{9}$ e três estudos não descreveram o sexo ${ }^{10-12}$.

No que se refere a faixa etária, três estudos apresentaram que a idade média dos 
conselheiros de saúde varia de 44 à $49 \operatorname{anos}^{7,9,12}$, dois estudos não citaram a idade ${ }^{8,11}$, um estudo encontrou em uma faixa de 50 à 60 anos $^{10}$ e uma outra pesquisa encontrou como resultado uma faixa etária de 28-53 anos para o sexo masculino e 33-48 para o feminino ${ }^{6}$. Desse modo, os resultados sugerem a falta de participação dos mais jovens em relação às questões políticas e de controle social. Além disso, tendo em vista que o representante do poder público é nomeado pela prefeitura, é comum que sejam indicadas pessoas mais experientes e com cargos elevados, que apresentem respaldo para debater junto à sociedade civil as demandas e reivindicações levantadas: no geral, estas são pessoas que se concentram em faixas etárias mais elevadas ${ }^{13}$.

Em relação ao nível de escolaridade dos conselheiros municipais observou-se que a maioria apresentava nível superior completo ${ }^{6,7,8,9,11}$, um estudo identificou uma tendência escalar, onde os representantes dos usuários entre o ensino fundamental e médio, os trabalhadores com nível superior e os membros da gestão e prestadores com superior completo ou pós-graduação ${ }^{10}$, um estudo apresentou que maioria não possuía ensino superior completo ${ }^{12}$.

De acordo com um dos estudos há uma tendência à elitização da participação nos conselhos, devido ao grau de instrução dos conselheiros ser, geralmente, mais alto do que o da média dos usuários do Sistema único de Saúde ${ }^{14}$.

\section{Conhecimento e Qualificação do Conselheiro Municipal de Saúde}

Em relação ao conhecimento dos Conselheiros Municipais de Saúde observou-se que quando perguntado aos conselheiros sobre o alcance dos objetivos do conselho, foi identificada uma assimilação de incompletude dos conselheiros, em grande parte, demonstrando falta de conhecimento ou dificuldades para expor com clareza suas opiniões em plenária. Segundo os entrevistados, parte dos assuntos que são discutidos no conselho é de grande complexidade para muitos dos envolvidos, ilustrando esse fato com a prestação de contas da secretaria. E se tratando desses assuntos, os próprios conselheiros compreendem que lhes falta capacitação para realizar a sua função ${ }^{7}$.

Contrapondo-se ao estudo anterior, os Conselheiros têm conhecimento das suas funções, porém, no discurso há a declaração de que alguns Conselheiros não estão atuando da forma adequada, pois exercem atribuições que não condizem com o Regimento, como por exemplo, a prática de ações assistenciais ${ }^{6}$. Desse modo, o conselho precisa rever sua maneira de atuar para que todos os seus integrantes tenham clareza de seu papel, ou seja, saibam exercer suas funções com confiança e convictos de que estarão buscando melhorias para toda a sociedade.

Alguns desafios encontrados no Conselho de Saúde foram identificados, tais como a falta de conhecimento dos conselheiros sobre seu papel no Conselho Municipal de Saúde e na esfera social e sanitária; o desconhecimento sobre as fontes de onde provêm as verbas que financiam o SUS; o desconhecimento do Regimento Interno e do Plano Municipal de Saúde, além da dificuldade de analisar o Relatório de Gestão ${ }^{11}$.

Outra pesquisa encontrou que $50 \%$ dos conselheiros de saúde relataram que há falta de informações importantes que poderiam auxiliar na melhora do seu desempenho, 70\% não sentem dificuldade em analisar documentos, relatórios e orçamentos, 54,8\% nunca participaram de discussão sobre orçamento e $35,7 \%$ e $22,2 \%$ relataram que os membros do seu conselho não entendem ou não são conscientes de suas tarefas. Desse modo, os conselhos são compostos por membros com pouco conhecimento sobre suas funções, grande parte relatou ter dificuldade na compreensão de documentos e o repasse de informações à sociedade e a discussão sobre orçamento são fatores a serem aprimorados ${ }^{12}$. Além disso, foram encontrados dois estudos que apresentam sobre a qualificação e capacitação dos conselheiros de saúde. Identificou-se que a maioria dos conselheiros não realizou nenhum tipo de qualificação ou capacitação específica, $40 \%$ disseram que participaram de palestras, congressos e seminários, e $6 \%$ não responderam. Desse modo, o autor sugere que a falta de qualificação ou capacitação para o exercício de conselheiro municipal de saúde pode ser considerada um problema, pois, para uma boa atuação no Conselho Municipal de Saúde, é necessário conhecer a estruturação do SUS e o papel dos conselhos de saúde ${ }^{7}$.

No que se refere à realização de qualificações, pode-se verificar que a maioria dos participantes $(62,5 \%)$ não as recebeu para exercer suas funções no Conselho ${ }^{9}$. Esse número expõe uma fragilidade que pode ser consequência da não oferta por parte dos gestores e dos próprios representantes de cada categoria, tanto na área do controle social quanto em outros temas, como informação e 
inclusão digital.

De acordo com os artigos analisados conclui-se que os conselhos municipais de saúde são compostos em sua maioria por homens, na faixa etária mais avançada e possui ensino superior completo, porém um estudo identificou que esse perfil é mais visualizado pelos gestores e profissionais de saúde. Além disso, a maioria dos estudos apresentaram a falta de conhecimento dos Conselheiros Municipais de Saúde sobre sua função.

\section{Referências}

1. Rocha EN, da Cunha JXP, Lira LSSP, Oliveira LB, Nery AA, Vilela ABA, Prado FO. O papel do conselheiro municipal de saúde na fiscalização do orçamento público. Saúde em Debate. 2013; 37(96): 104-111.

2. Brasil. Casa Civil. Lei no 8.080, de 19 de setembro de 1990. Dispõe sobre as condições para a promoção, proteção e recuperação da saúde, a organização e o funcionamento dos serviços correspondentes, e dá outras providências. Diário Oficial da União, Brasília, DF, 20 set. 1990 a.

3. dos Santos $\mathrm{MH}$. A importância do controle social para o desenvolvimento de adequadas políticas públicas em saúde no município de Anápolis-GO. Revista Jurídica. 2013; 1: 05-12.

4. Cotta RMM, Mendes FF, Muniz JN. Descentralização das políticas públicas de saúde: do imaginário ao real. Viçosa: UFV/CEBES, 1998. $148 p$.

5. da Silva AX, Cruz EA, Melo VA. Importância estratégica da informação em saúde para o exercício do controle social. Cienc Saude Colet. 2007; 12(3):683-688.

6. Souza TO, Silva MJ, Nobrega SS, Constancio JF. Controle social: um desafio para o conselheiro de saúde. Rev. bras. enferm. [online]. 2012; 65(2): 215-221.

$7 . \quad$ Lemos CLS, Bovo KLO, Costa JB, Silveira NA, Cardoso CG. Perfil e visão dos conselheiros municipais de saúde sobre sua atuação. Rev. Gest.Saúde. 2016; 07(03): 1126-39.

8. Ramos, MF, Cezare, JP, Vendramini PRJ, Coutinho SMV, Reis, TS, Fernandes V. Conselhos setoriais: perfil dos conselheiros e sua influência na tomada de decisão / Sector Councils: councilors' profile and it's influence in decision making. Saúde Soc. 2012; 21: 61-70.
9. Fernandes VC, Spagnuolo RS, Nascimento EM. Percepção de conselheiros de saúde sobre acesso às informações e inclusão digital. Saúde Soc. 2017; 26 (1): 218-228.

10. Lopes BJ, Silva EA, Martins S. Conselho Municipal de Saúde sob a ótica de seus conselheiros: estudo realizado em uma capital brasileira. Interações. 2016; 17 (2): 163-172.

11. Cotta, RMM, Cazal, MM, Rodrigues JFC. Participação, Controle Social e Exercício da Cidadania: a (des)informação como obstáculo à atuação dos conselheiros de saúde. Physis. 2009; 19(2): 419-438.

12. Saliba NA, Moimaz SAS, Ferreira NF, Custódio LBM.*. Conselhos de saúde: conhecimento sobre as ações de saúde. Rev $A D M$ Publica (RAP). 2009; 43(6):1369-1378.

13. Cezare JP. Conselhos municipais $e$ governança: uma análise do Conselho de Representantes de Paranapiacaba e Parque Andreense do Município de Santo André - SP. 2009. Dissertação (Mestrado em Saúde Pública) Faculdade de Saúde Pública da Universidade de São Paulo, São Paulo.

14. Stralen CJV, Lima AMD, Sobrinho DF, Saraiva LDES, Stranlen TBDSV, Belisário SA. Conselhos de saúde: efetividade do controle em municípios de Goiás e Mato Grosso do Sul. Cien Saude Colet. 2006; 11(3): 621-632.

\section{Endereço para Correspondência}

Universidade Estadual de ponta Grossa - UEPG

Estrada do Bem Querer, km 4, Caixa Postal 95. Vitória da Conquista - BA

CEP.: 45083-900

e-mail: rafaelspassos08@gmail.com

Recebido em 09/02/2018

Aprovado em 19/06/2018

Publicado em 20/12/2018 